\title{
Intervertebral disc degeneration associated with vertebral marrow fat, assessed using quantitative magnetic resonance imaging
}

\author{
Yayun $\mathrm{Ji}^{1} \cdot$ Weifeng Hong ${ }^{1} \cdot$ Mouyuan Liu ${ }^{1}$ Y Yuying Liang ${ }^{1} \cdot$ YongYan Deng ${ }^{1} \cdot$ Liheng Ma $^{1}$
}

Received: 28 December 2019 / Revised: 10 March 2020 / Accepted: 10 March 2020 / Published online: 28 May 2020

(C) The Author(s) 2020

\begin{abstract}
Objective To investigate the potential clinical application of quantitative MRI in assessing the correlation between lumbar vertebrae bone marrow fat deposition and intervertebral disc degeneration.

Materials and methods A total of 104 chronic lower-back pain volunteers underwent 3.0-T MRI with T2-weighted imaging, T2 mapping, and iterative decomposition of water and fat with echo asymmetry and least squares estimation (IDEAL-IQ) between August 2018 and June 2019. Each disc was assessed with T2 value by T2 mapping, and the L1-S1 vertebral bone marrow fat fraction was assessed by IDEAL-IQ. The differences and relationship between T2 value and the adjacent vertebral bone marrow fat fraction values within the five Pfirmann groups, five age groups, and five lumbar levels were statistically analyzed.

Results The vertebral bone marrow fat fraction had a significant negative correlation with $\mathrm{T} 2$ values of nucleus pulposus' $\mathrm{T} 2$ values $(p<0.001)$. However, the significant negative correlation was only found between $\mathrm{T} 2$ values of nucleus pulposus and adjacent vertebral bone marrow fat in Pfirrmann II-III, L1/2-L5/S1 level, and 40-49 years' age groups. Pfirrmann grades of the intervertebral disc were positively correlated with adjacent vertebrae bone marrow fat fraction $(p<0.05)$.

Conclusion Lumbar bone marrow fat deposition significantly increases during the early stages of intervertebral disc degeneration. Quantitative measurements of bone marrow fat deposition and water content of intervertebral discs have a predictive value and are an important supplement to the qualitative traditional classification strategies for the early stages of intervertebral disc degeneration.
\end{abstract}

Keywords Lumbar disc degeneration · Quantitative MRI · Bone marrow fat

\begin{tabular}{|c|c|c|c|}
\hline & FF & Fat fraction \\
\hline \multirow{4}{*}{$\begin{array}{l}\text { QMRI } \\
\text { IDEAL-IQ }\end{array}$} & Quantitative magnetic resonance imaging & VFF & Vertebral fat fraction (bone marrow \\
\hline & Iterative decomposition of water & & fat fraction of the vertebra) \\
\hline & and fat with echo asymmetry & SFF & Superior vertebral fat fraction \\
\hline & and least squares estimation & IFF & Inferior vertebral fat fraction \\
\hline $\mathrm{BMF}$ & Bone marrow fat & NP & Nucleus pulposus \\
\hline IVD & Intervertebral disc & AAF & Anterior annulus fibrosus \\
\hline IVDD & Intervertebral disc degeneration & PAF & Posterior annulus fibrosus \\
\hline
\end{tabular}

Yayun Ji and Weifeng Hong contributed equally to this work.

Liheng Ma

maliheng@gdpu.edu.com

Yayun Ji

jiyayun@163.com

Weifeng Hong

hongweifeng413@163.com

Mouyuan Liu

1350504757@163.com
Yuying Liang

yuying_liang@126.com

YongYan Deng

yongyandeng999@163.com

1 Department of Medical Imaging, The First Affiliated Hospital of Guangdong Pharmaceutical University, Guangzhou City 510080, Guangdong Province, China 
Table 1 Classification of disc degeneration [25]

\begin{tabular}{lllll}
\hline $\begin{array}{l}\text { Pfirrmann } \\
\text { classification }\end{array}$ & Structure & Nucleus and anulus & Signal intensity & $\begin{array}{l}\text { Height of } \\
\text { intervertebral disc }\end{array}$ \\
\hline I & Homogeneous, bright white & Clear & $\begin{array}{c}\text { Hyperintense, isointense to } \\
\text { cerebrospinal fluid } \\
\text { Hyperintense, isointense to } \\
\text { cerebrospinal fluid }\end{array}$ & Normal \\
II & $\begin{array}{l}\text { Inhomogeneous with or } \\
\text { without horizontal bands }\end{array}$ & Clear & Intermediate & Normal to slightly decreased \\
III & $\begin{array}{l}\text { Inhomogeneous, gray } \\
\text { Inhomogeneous, gray to black }\end{array}$ & Lost & Intermediate to hypointense & Normal to moderately decreased \\
V & Inhomogeneous, black & Lost & Hypointense & Collapsed disc space \\
\hline
\end{tabular}

\section{Introduction}

Lumbar intervertebral disc degeneration (IVDD) is a common orthopedics' disease that causes lower-back pain [1-3], with early biochemical changes which include proteoglycans and hydration loss, and late morphologic changes which include disc height loss, nucleus pulposus (NP) herniation, and annular tears [4]. The lumbar disc is the largest avascular tissue within the human body, nourished primarily by the diffusion of micro-vessels in the adjacent vertebral bodies through the cartilaginous endplate [5].
Interference with this pathway may be a risk factor for intervertebral disc (IVD) metabolism. The conversion of the hematopoietic bone marrow to bone marrow fat (BMF) accompanies the decrease of vertebral perfusion and nutrition supplies [6,7]. However, whether the adjacent vertebra marrow fat can affect IVDD remains unknown.

Osteoporosis is closely associated with IVDD [8] while vertebral osteoporosis is associated with IVDD in postmenopausal women [9]. IVDD is often concomitant with osteoporosis $[10,11]$, suggesting that osteoporosis and IVDD development may be an accompanying process and may explain

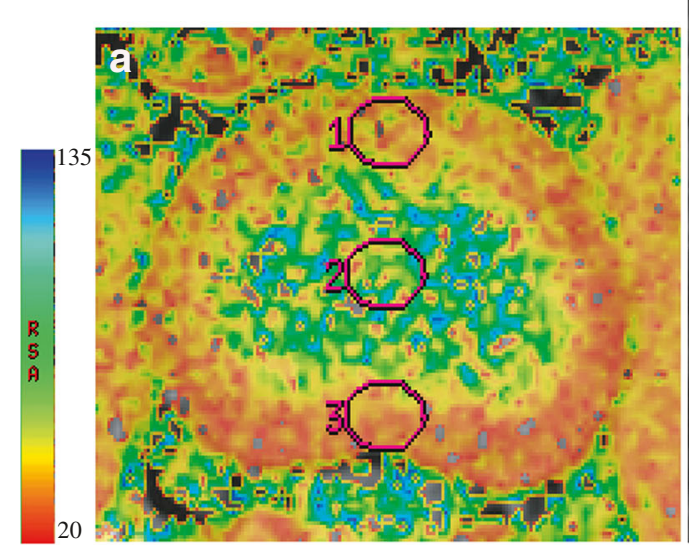

Fig. 1 T2WI was used to assess the Pfirrmann classification. The ROIs were drawn in the axial T2 mapping images referred to the sagittal view to measure the T2 values of nucleus pulposus (NP), anterior annulus fibrosus (AAF), and posterior annulus fibrosus (PAF) separately $(1=$

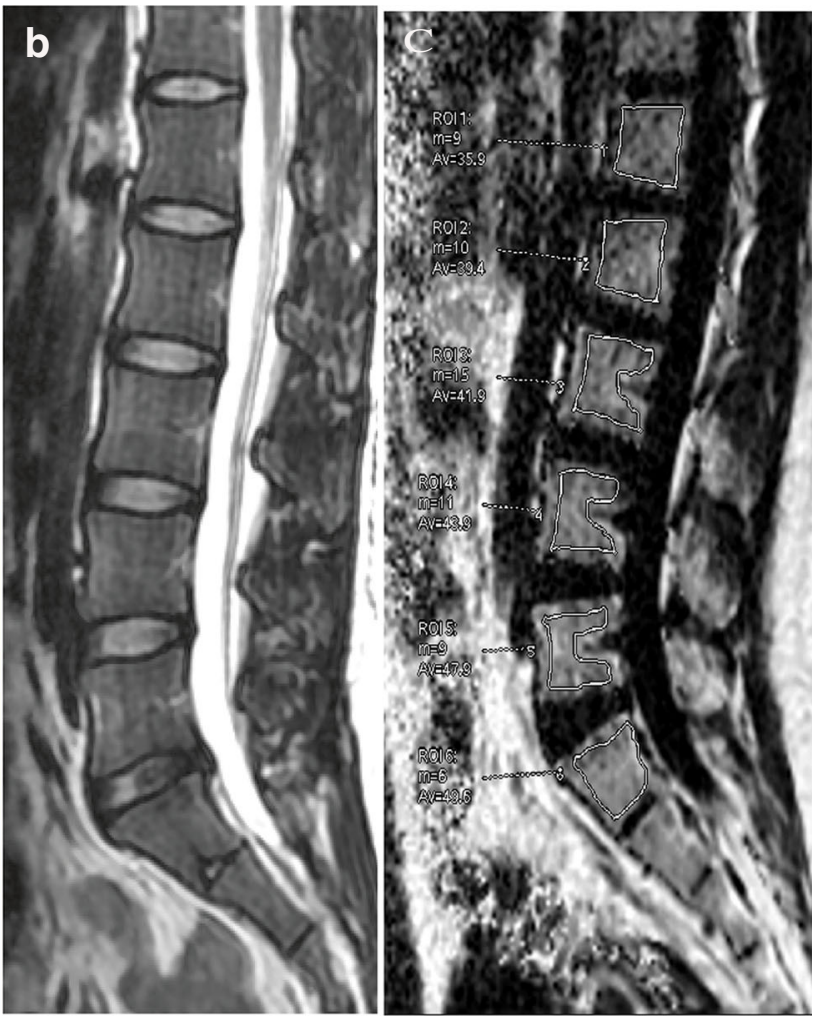

AAF, $2=\mathrm{NP}, 3=\mathrm{PAF}$ ). The vertebral bone marrow fat was measured with ROI in vertebrae, in which the endplate and basivertebral foramen were avoided 
Table 2 Participants' characteristics

\begin{tabular}{ll}
\hline Characteristics & Number of cases $(\%)$ \\
\hline Gender & \\
Male & $47(45.2)$ \\
Female & $57(54.8)$ \\
BMI $\left(\mathrm{kg} / \mathrm{m}^{2}\right)$ & 23.2 \\
Intervertebral disc & 520 \\
Lumbar vertebral body & 624 \\
Pfirrmann grading & \\
I & $196(38.7)$ \\
II & $140(26.9)$ \\
III & $95(18.3)$ \\
IV & $66(12.7)$ \\
V & $23(4.4)$ \\
Age (years) & \\
$20-29$ & $27(26.0)$ \\
$30-39$ & $17(16.3)$ \\
$40-49$ & $17(16.3)$ \\
$50-59$ & $26(25.0)$ \\
$60-70$ & $17(16.3)$ \\
\hline
\end{tabular}

why postmenopausal women experience more back pain than men [12]. Studies have reported the possibility of delaying IVDD progression by improving bone metabolism and vertebral osteoporosis. For example, sodium alendronate delays the progression of lumbar IVDD in ovariectomized rats by improving bone quality [13].

Folman et al. showed that bone marrow density (BMD) positively correlated with disc degeneration [14]. One explanation is that most studies measure BMD using dual-energy X-ray absorptiometry (DXA). Since the DXA measurement of BMD often interferes with the results due to osteophyte formation, it may not accurately reflect the degree of bone changes in the vertebral body. Other non-invasive methods are required to accurately detect and explore vertebral BMD changes.

Vertebral BMF deposition is negatively associated with bone density and closely related to osteoporosis $[15,16]$. During osteoporosis, the differentiation programs that yield osteoblasts and adipocytes from mesenchymal stem cells within the marrow favor adipogenesis to osteoblastogenesis leading to increased marrow fat with reduced bone formation [17-19]. Various advanced MRI techniques have been used to measure BMF content, and IDEAL-IQ was confirmed as one of the most
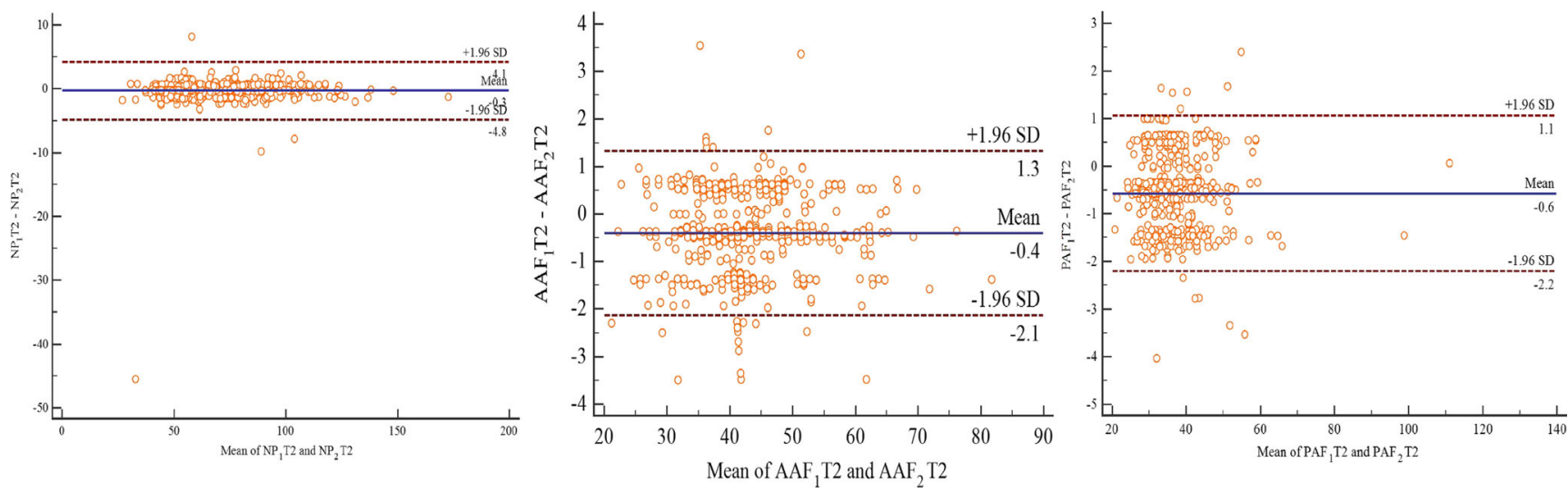

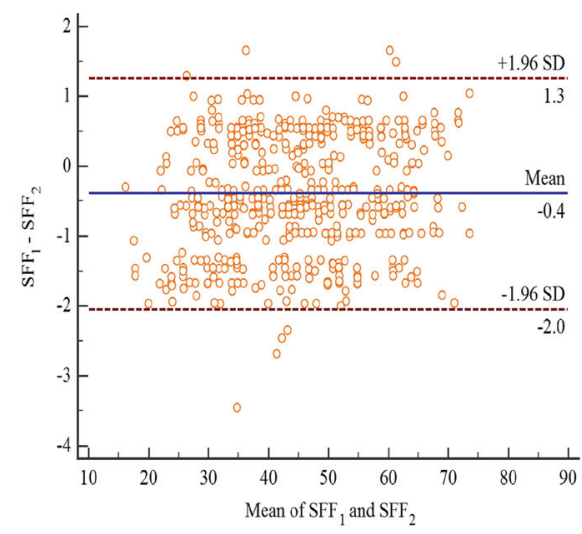

Fig. 2 Bland-Altman plots comparing all paired measurements, including NP, AAF, PAF, SFF, and IFF. Bias (solid line) and limits of agreement (dashed line) are shown for each variable. The mean score is plotted on the $x$-axis, while the difference between the two devices is

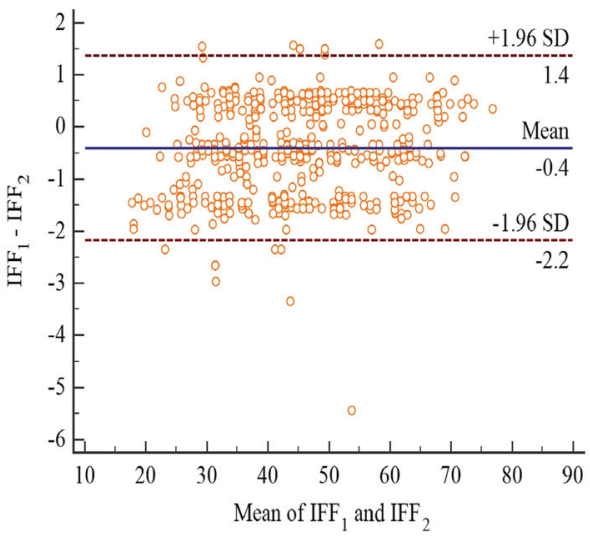

plotted on the $y$-axis (mean difference $\pm 1.96 \mathrm{SD}$ ). SFF, superior vertebral fat fraction; IFF, inferior vertebral fat fraction; NP, nucleus pulposus; AAF, anterior annulus fibrosus; PAF, posterior annulus fibrosus 


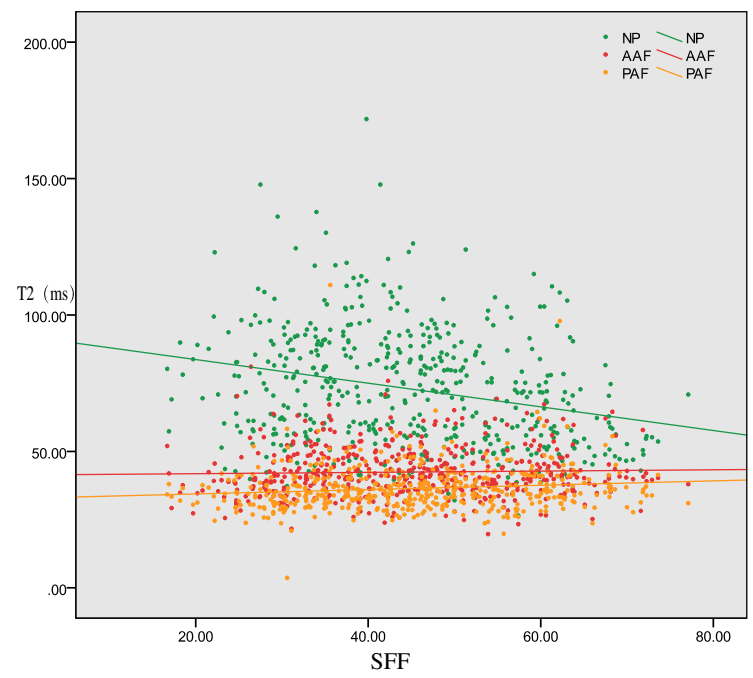

Fig. 3 Correlation between all the T2 values of annulus fibrosus and nucleus pulposus and the FF values of adjacent superior and inferior vertebrae together. The T2 values of the NP and AAF and PAF significantly correlated with the FF values of the adjacent superior and

convenient and accurate techniques to quantify BMF [20,21]. T2 mapping, a novel quantitative technique, evaluates disc degeneration. Compared with traditional Pfirrmann classification, T2 mapping is less affected by subjective factors, and the measurement of disc degeneration is more accurate [22, 23]. This study investigated whether BMF parameters correlate with IVDD at different ages by quantitative MRI.

\section{Materials and methods}

\section{Study population}

This cross-sectional cohort study was approved by the Ethics Committee of our hospital. All subjects provided written informed consent before examination. A total of 104 male and female volunteers of different age groups (20-29 years, 3039 years, $40-49$ years, $50-59$ years, $60-70$ years) with chronic lower-back pain between August 2018 and June 2019 were recruited in this study. Among them, 47 were male (mean age 44.6 years) and 56 were female (mean age 44.3 years). The inclusion criteria included the following: (1) male or female volunteers with chronic lower-back pain (lasted for more than 3 months), which was based on Alleva et al.'s study [24]; (2) underwent routine MRI lumbar spine examination, IDEAL-IQ, and T2 mapping imaging with satisfactory image quality. Exclusion criteria included the following: (1) medical history of diseases, such as a tumor, spondylolisthesis, lumbar scoliosis, lumbar trauma or fracture, surgery, spinal infectious diseases, hemopathy, and metabolic bone disease; (2) incomplete imaging data or poor image quality.

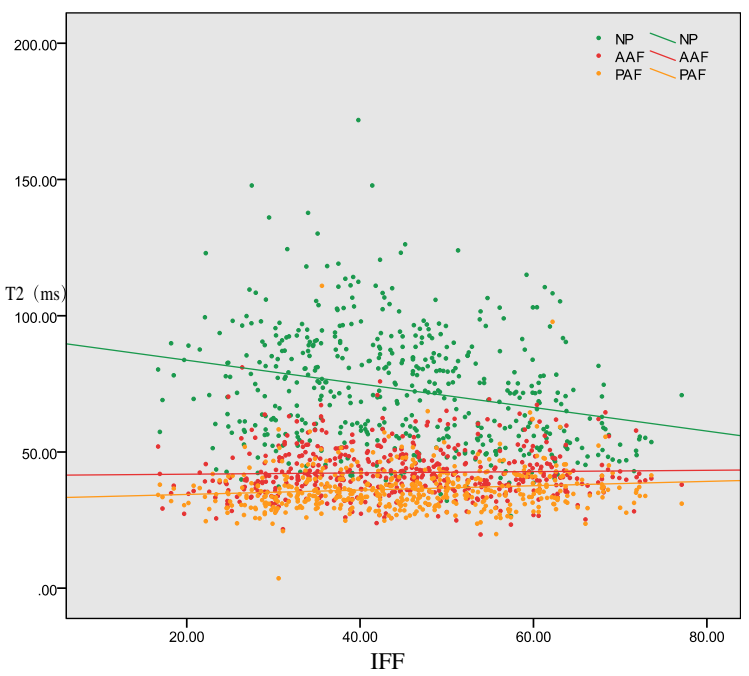

inferior vertebral bodies $(p<0.001)$. FF, fat fraction; SFF, superior vertebral fat fraction; IFF, inferior vertebral fat fraction; NP, nucleus pulposus; AAF, anterior annulus fibrosus; PAF, posterior annulus fibrosus

\section{MRI imaging protocol}

MRI was performed using a 3T system (GE Discovery MR750 3.0T, America) with a dedicated spine coil. The lumbar spine scanning sequence included routine T1WI and T2WI sequence, IDEAL-IQ, and T2 mapping sequence. The following imaging parameters were used: (1) Sagittal T1WI (TR/TE $=809 / 11 \mathrm{~ms}$ ), $\mathrm{T} 2 \mathrm{WI}(\mathrm{TR} / \mathrm{TE}=3093 / 120 \mathrm{~ms})$, transversal T2WI $(\mathrm{TR} / \mathrm{TE}=$ $3500 / 120 \mathrm{~ms}$ ), slice thickness $3 \mathrm{~mm}$, intersection gap 0.4 , FOV $320 \times 320 \mathrm{~mm}$, matrix $132 \times 132$, the number of excitations (NEX) 2; (2) Sagittal IDEAL-IQ:TR $10.5 \mathrm{~ms}$, minimum TE $1.2 \mathrm{~ms}$, maximum TE $6.5 \mathrm{~ms}$, FOV $300.0 \mathrm{~mm} \times 300.0 \mathrm{~mm}$, matrix $132 \times 132$, layer thickness $5.0 \mathrm{~mm}$, acquisition time $168 \mathrm{~s}$; (3) transversal T2 mapping TR $718 \mathrm{~ms}$, TE1-TE8 time $9.7 \mathrm{~ms}, 19.4 \mathrm{~ms}, 29.1 \mathrm{~ms}, 38.7 \mathrm{~ms}, 48.4 \mathrm{~ms}, 58.1 \mathrm{~ms}, 67.8 \mathrm{~ms}$, and $77.5 \mathrm{~ms}$, layer thickness $3 \mathrm{~mm}$, layer spacing $1 \mathrm{~mm}$, NEX 1 .

\section{Quantitative MR image analysis}

All routine MR images were used to identify pre-existing abnormalities of the lumbar vertebrae, and T2WI was used to assess the Pfirrmann classification [25]; the specific classification standards are listed in Table 1; we excluded 23 Pfirrmann V IVDs. All vertebral bone marrow fat fractions (FF) were measured along with the remaining 497 Pfirrmann I-IV IVDs' T2 values on the AW4.6 workstation. All MRI images were analyzed by two experienced radiologists in clinical diagnostic and spine MRI research, MLH, and JYY. We loaded FatFrac images in the REFOMAT system and measured the FF value of the vertebral bone marrow (VFF). The L1-S1 vertebral body was mapped manually at the three central layers, to avoid the endplate and basivertebral foramen. To 
avoid bias, the average value of the three measurements was considered (Fig. 1). We defined the fat fraction of adjacent superior vertebral bodies as SFF and the fat fraction of adjacent inferior vertebral bodies as IFF. The Functool postprocessing was also performed on the T2 mapping images. We indicated the round region of interesting (ROI) within the anterior annulus fibrosus (AAF) to measure the T2 value and copied the ROI onto the nucleus pulposus (NP) and posterior annulus fibrosus (PAF) to measure the $\mathrm{T} 2$ values, respectively (Fig. 1). The T2 values of the NP, AAF, and PAF of L1/2-L5/S1 discs were all measured. The final Pfirrmann results were obtained by consensus of the two experts, and the average value of the two expert measurements was taken for the quantitative result.

\section{Statistical analysis}

Agreement between the two experts' Pfirrmann gradings was evaluated using Cohen's kappa $(\kappa)$ statistic. The strength classification based on kappa values was as follows: excellent $(\kappa=0.8-1.0)$; substantial $(\kappa=0.6-0.80)$; moderate $(\kappa=0.4$ $0.6)$; fair $(\kappa=0.2-0.4)$; and slight $(\kappa=0.0-0.2)$. A BlandAltman plot was used to analyze the quantitative measurements between the two experts, by plotting the mean of the two measurements against their difference with a $95 \%$ limits of agreement $(\mathrm{LOA})(=$ mean difference $\pm 1.96 \times \mathrm{SD}$ of the difference). Bivariate correlations of the T2 value, Pfirrmann $\mathrm{I}-\mathrm{V}$ in IVD, and FF in the adjacent vertebra were measured using the Pearson product-moment two-tailed correlation
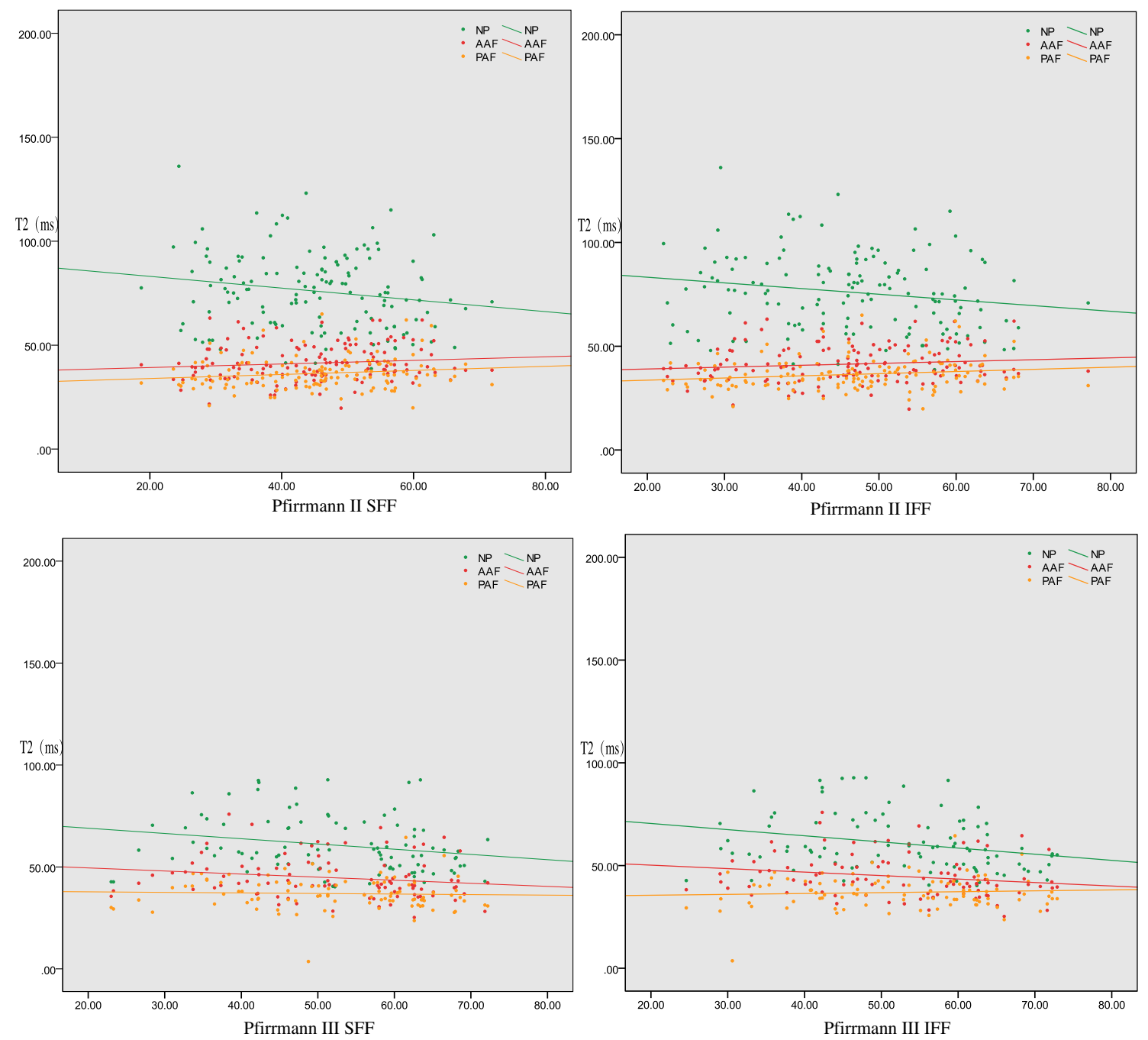

Fig. 4 Correlation between the T2 values of annulus fibrosus and nucleus pulposus in different Pfirrmann grades and the FF values of adjacent superior and inferior vertebrae. The T2 values of the NP of grade II and III significantly correlated with the FF values of the adjacent superior and inferior vertebral bodies $(p<0.05)$, while the other T2 values of the NP

and annulus fibrosus did not significantly correlate with the FF values of the adjacent upper and lower vertebral bodies $(p>0.05)$. FF, fat fraction; $\mathrm{SFF}$, superior vertebral fat fraction; IFF, inferior vertebral fat fraction; $\mathrm{NP}$, nucleus pulposus; $\mathrm{AAF}$, anterior annulus fibrosus; PAF, posterior annulus fibrosus 

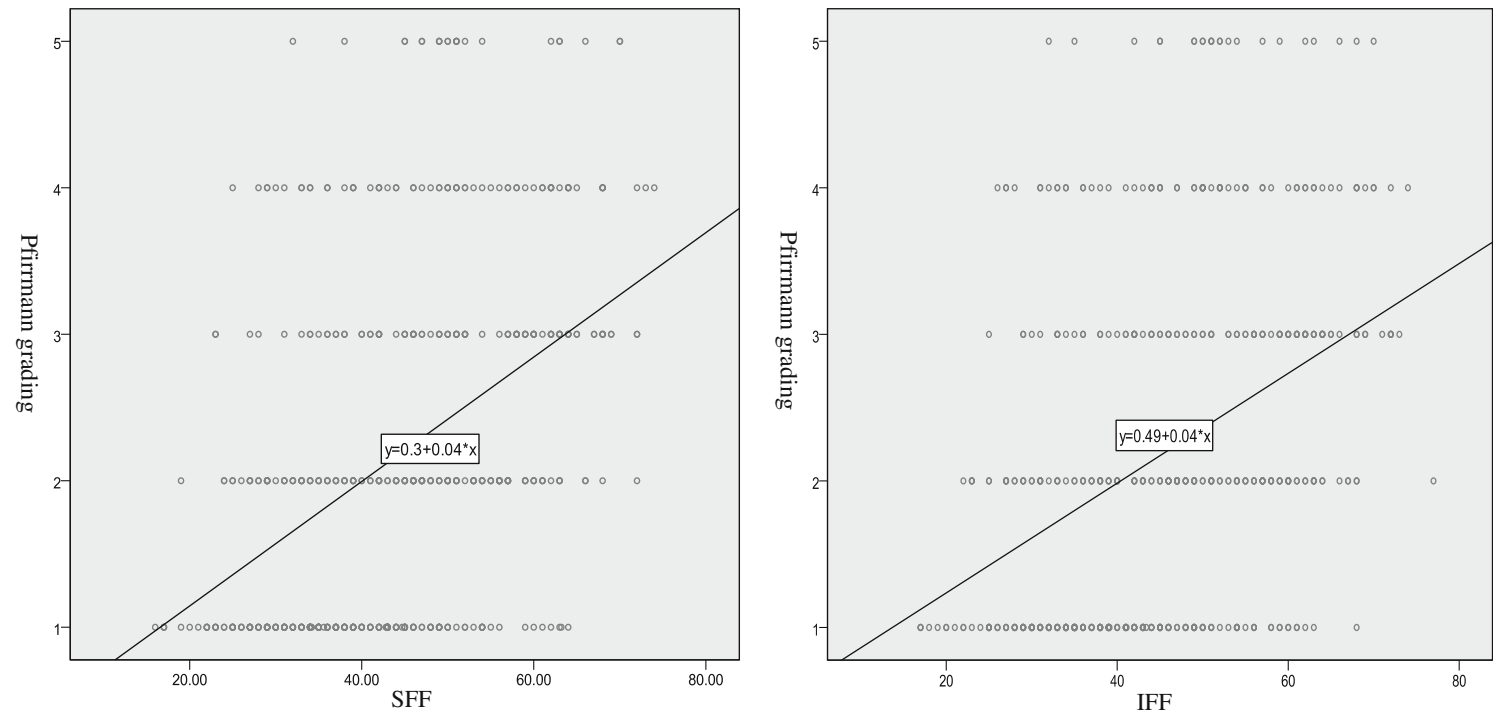

Fig. 5 Different Pfirrmann grades of intervertebral discs correlated significantly with VFF values in the superior vertebrae and inferior vertebrae $(p<0.01)$. VFF, vertebral fat fraction; SFF, superior vertebral fat fraction; IFF, inferior vertebral fat fraction

coefficient analysis. The $\mathrm{T} 2$ values and the adjacent $\mathrm{SFF} / \mathrm{IFF}$ value differences within the five Pfirrmann groups, five age groups, and five lumbar levels were examined using the Kruskal-Wallis test [26]. Analyses, where the overall test was significant, and pairwise comparisons were carried out using the Mann-Whitney $U$ test while controlling for type I error using the Bonferroni adjustment. Data were represented as boxplots. All statistical analyses were performed using the SPSS version 23 (IBM, Chicago, IL, USA). $p<0.05$ was considered statistically significant.

\section{Results}

\section{Study population}

In total, 520 IVDs were analyzed from the 104 volunteers. The mean body mass index (BMI) was $23.2 \pm 3.2 \mathrm{~kg} / \mathrm{m}^{2}$ (ranged 16.2-33.2), and there were 196 (38.7\%), 140 (26.9\%), 95 $(18.3 \%), 66(12.7 \%)$, and $23(4.4 \%)$ IVDs in each of the Pfirrmann classification from grade I to $\mathrm{V}$, respectively
(Table 2). The remaining 497 IVDs of Pfirrmann I-IV were quantitatively analyzed.

Pfirrmann classification of $\kappa=0.83$ demonstrated an excellent agreement, while Bland-Altman analysis of all paired measurements revealed a mean difference $( \pm \mathrm{SD}, 95 \%$ limits of agreement) of $-0.3 \mathrm{~ms}$ ( $\pm 2.3 \mathrm{~ms},-4.8$ to $4.1 \mathrm{~ms})$ for NP; $-0.4 \mathrm{~ms}$ ( \pm $0.9 \mathrm{~ms},-0.5$ to $-0.3 \mathrm{~ms})$ for AAF; $-0.6 \mathrm{~ms}( \pm 0.8 \mathrm{~ms},-0.7$ to $-0.5 \mathrm{~ms})$ for PAF; $-0.4 \mathrm{~ms}( \pm 0.8 \mathrm{~ms},-0.5$ to $-0.3 \mathrm{mmHg})$ for $\mathrm{SFF}$; and $-0.4 \mathrm{~ms}( \pm 0.9 \mathrm{~ms},-0.5$ to $-0.3 \mathrm{mmHg})$ for IFF as shown in Fig. 2.

\section{Correlations of VFF and T2 in AAF, NP, PAF/ Pfirrmann grading in all discs, and lumbar vertebral bodies}

For all 497 discs, the T2 value of the NP/AAF/PAF significantly correlated with not only the SFF but also the IFF $(p<0.001$, Fig. 3$)$. The NP T2 was negatively correlated with adjacent VFF ( $p<0.001$, Fig. 4). The Pfirrmann classification I-V correlated positively with the adjacent SFF and IFF $(p<0.01)$ (Fig. 5).
Table 3 Differences of VFF and $\mathrm{T} 2$ in $\mathrm{AAF}, \mathrm{NP}$, and PAF in the four Pfirrmann groups

\begin{tabular}{llllll}
\hline Pfirrmann grade & \multicolumn{2}{l}{ T2 } & & SFF & IFF \\
\cline { 2 - 4 } & NP & AAF & PAF & & \\
\hline I & $83.9 \pm 20.5$ & $42.5 \pm 8.7$ & $36.0 \pm 9.5$ & $37.1 \pm 9.8$ & $38.4 \pm 10.3$ \\
II & $76.2 \pm 19.1$ & $41.6 \pm 8.8$ & $36.6 \pm 7.5$ & $45.0 \pm 11.3$ & $46.3 \pm 12.0$ \\
III & $60.6 \pm 13.2$ & $44.4 \pm 10.1$ & $37.0 \pm 7.7$ & $52.1 \pm 11.8$ & $52.5 \pm 12.0$ \\
IV & $52.2 \pm 10.2$ & $41.1 \pm 9.2$ & $36.2 \pm 6.7$ & $50.5 \pm 12.7$ & $49.9 \pm 12.7$ \\
$\chi 2$ & 161.983 & 7.048 & 4.291 & 111.970 & 93.933 \\
$p$ & 0.000 & 0.070 & 0.0232 & 0.000 & 0.000 \\
\hline
\end{tabular}

$S F F$, superior vertebral fat fraction; $I F F$, inferior vertebral fat fraction; $N P$, nucleus pulposus; $A A F$, anterior annulus fibrosus; $P A F$, posterior annulus fibrosus 
Table 4 Differences of II-III and IV-V in Mann-Whitney $U$ test

\begin{tabular}{|c|c|c|c|c|c|c|c|c|}
\hline \multirow[t]{2}{*}{ II-III vs IV } & \multicolumn{3}{|l|}{$\mathrm{T} 2$} & \multirow[t]{2}{*}{ SFF } & \multirow[t]{2}{*}{ IFF } & \multirow[t]{2}{*}{ Ages } & \multirow[t]{2}{*}{ Gender } & \multirow[t]{2}{*}{ BMI } \\
\hline & NP & $\mathrm{AAF}$ & PAF & & & & & \\
\hline $\mathrm{Z}$ & -7.264 & -1.044 & -0.565 & -1.496 & -0.585 & -1.714 & -0.065 & -0.962 \\
\hline$p$ & 0.000 & 0.297 & 0.572 & 0.135 & 0.559 & 0.086 & 0.948 & 0.336 \\
\hline
\end{tabular}

$S F F$, superior vertebral fat fraction; $I F F$, inferior vertebral fat fraction; $N P$, nucleus pulposus; $A A F$, anterior annulus fibrosus; $P A F$, posterior annulus fibrosus

\section{Correlations and differences of VFF and T2 in AAF, NP, PAF/Pfirrmann grading in the four Pfirrmann groups}

There was no statistical difference between SFF and IFF adjacent to all the IVDs $(p>0.05)$. However, only the T2 values of NP significantly inversely correlated with the adjacent VFF in Pfirrmann classification degrees II-III but not in grades IVV (Fig. 3). When comparing II-III and IV parameters, significant differences were observed in the NP T2 values and SFF, but none was found in the AAF T2, PAF T2, adjacent IFF, ages, gender, and the BMI parameters (Tables 3 and 4). NP T2 values decreased gradually from Pfirmann I to IV grades, while FF values increased in Pfirrmann I-III and decreased in Pfirrmann IV (Fig. 6).

\section{Correlations and differences of VFF and T2 in AAF, NP, and PAF/Pfirrmann grading in the five age groups}

Quantitative MRI characteristics were assessed for age groups: 20-29 years, 30-39 years, 40-49 years, 50-59 years, and 60-70 years (Fig. 7). T2 values of NP were weakly correlated with the adjacent VFF values in the age group 40-
49 years $(r=0.246)$. The VFF value increased with age while the NP T2 value decreased after the age of 30 . After the age of 50 , VFF increases more rapidly. The NP T2 value and VFF of adjacent vertebrae bone were significantly different between the age groups 30-39 and 40-49, and the age groups 50-59 and $60-70(p<0.001)$ (Table 5).

\section{Correlations and differences of VFF and T2 in AAF, NP, and PAF/Pfirrmann grading in the five lumbar levels}

We observed a relationship not only between the NP T2 value and adjacent VFF but also between the Pfirrmann grading and adjacent VFF in L1/2-L5/S1 levels, which was statistically significant except for the L5/S1 NP T2 value and the S1 VFF. However, the AAF and PAF did not significantly correlate to VFF (Figs. 8 and 9). T2 value of NP, AAF, and PAF and its adjacent SFF were significantly difference from those of L1/2 to L5/S1 (Table 6). Among the 104 volunteers, the number of IVDD (Pfirmann II-V IVD) occurring alone in one segment was 8 and contained the following: $\mathrm{L} 1 / 2(n=$ $0), \mathrm{L} 2 / 3(n=0), \mathrm{L} 4 / 5(n=4), \mathrm{L} 3 / 4(n=2)$, and L5/S1 $(n=2)$.

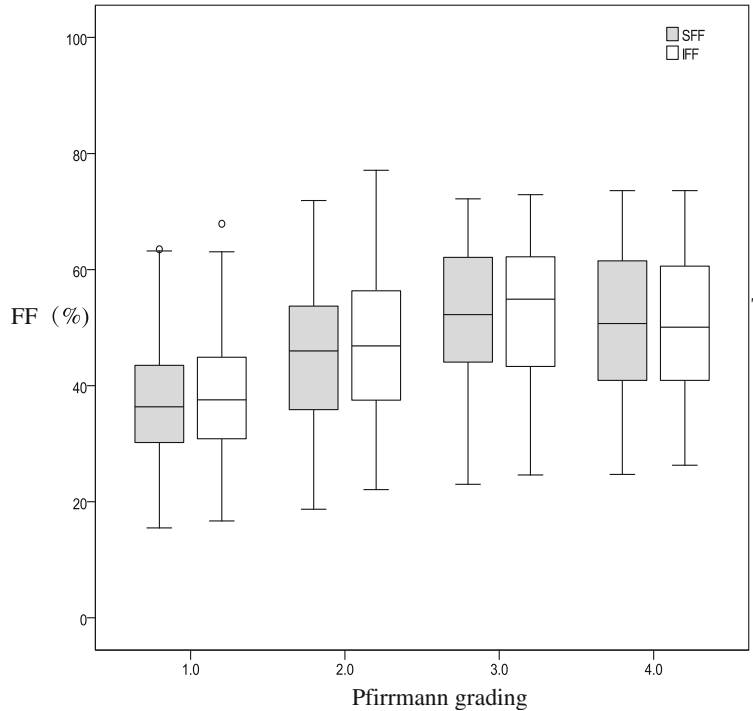

Fig. 6 Box chart of different Pfirmann-graded disc NP and annulus fibrosus T2 values of the upper and lower adjacent vertebral body FF values. NP T2 values are decreasing gradually but FF values are inversely

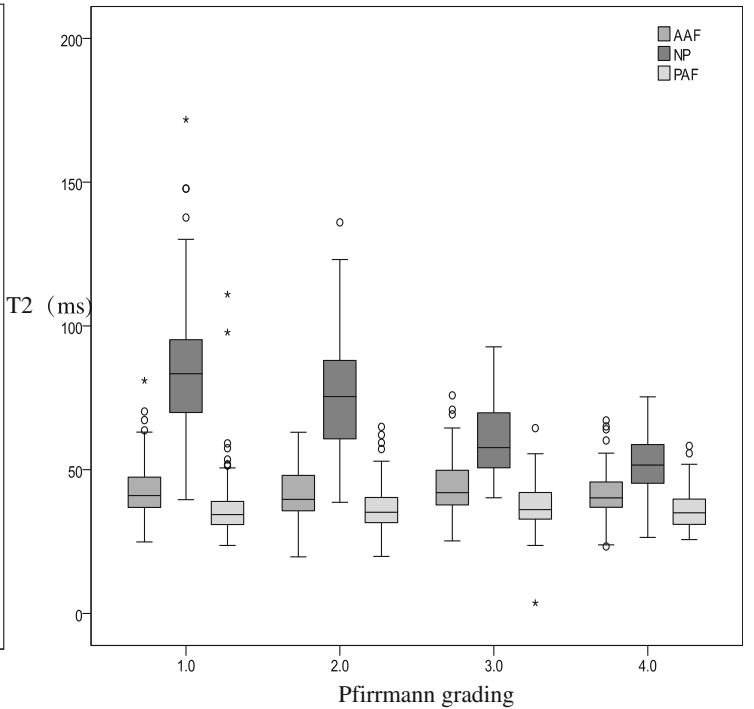

increasing only in Pfirrmann I-III. FF, fat fraction; SFF, superior vertebral fat fraction; IFF, inferior vertebral fat fraction; NP, nucleus pulposus; $\mathrm{AAF}$, anterior annulus fibrosus; $\mathrm{PAF}$, posterior annulus fibrosus 


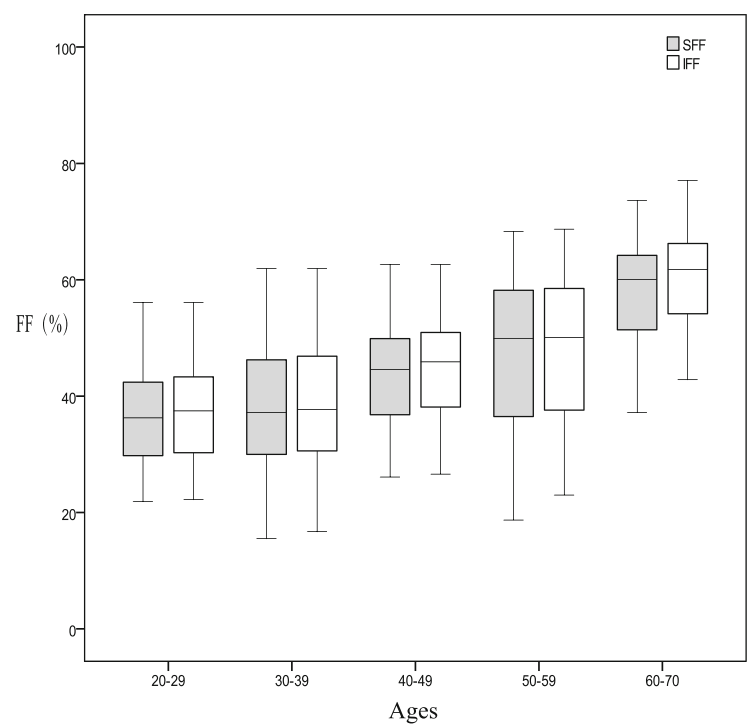

Fig. $7 \mathrm{FF}$ values of the vertebral body increased with age, while the T2 value of the NP decreased after age 30 . After age 50, FF increases more rapidly than normal. $\mathrm{FF}$, fat fraction; $\mathrm{SFF}$, superior vertebral fat fraction;

\section{Discussion}

We investigated whether IVDD is associated with adjacent vertebrae BMF using advanced quantitative MRI techniques. We found clear correlation between the T2 of NP/AAF/PAF and adjacent VFF when we put all the intervertebral discs and vertebral bodies together. Furthermore, according to the Pfirrmann classification standard, we proposed grade I as representing a normal disc, grade II and III as representing the early stages of disc degeneration, and grade IV as representing the advanced stages of disc degeneration. The NP T2 values of early degenerated IVD (grade II-III) are greater than those advanced (grade IV). Our study revealed that only the NP T2 value of early degenerative disc correlated mildly with the adjacent vertebrae BMF content and differed significantly

Table 5 Differences of VFF and T2 in AAF, NP, and PAF in the five age groups

\begin{tabular}{llllll}
\hline \multirow{2}{*}{ Ages } & T2 & \multicolumn{5}{c}{ SFF } & IFF \\
\cline { 2 - 4 } & NP & AAF & PAF & & \\
\hline $20-29$ & $80.9 \pm 22.9$ & $41.0 \pm 8.1$ & $33.4 \pm 5.1$ & $36.2 \pm 7.7$ & $36.9 \pm 7.6$ \\
$30-39$ & $86.6 \pm 20.0$ & $43.3 \pm 9.6$ & $38.5 \pm 10.0$ & $37.8 \pm 11.6$ & $38.3 \pm 11.8$ \\
$40-49$ & $68.0 \pm 17.8$ & $40.6 \pm 8.2$ & $35.1 \pm 6.0$ & $44.0 \pm 9.2$ & $45.1 \pm 9.1$ \\
$50-59$ & $67.5 \pm 17.1$ & $45.0 \pm 10.0$ & $38.6 \pm 10.2$ & $47.9 \pm 12.0$ & $48.6 \pm 12.2$ \\
$60-70$ & $57.1 \pm 12.4$ & $42.0 \pm 9.2$ & $37.2 \pm 7.8$ & $58.6 \pm 8.9$ & $60.3 \pm 8.4$ \\
$\chi 2$ & 110.852 & 14.379 & 41.747 & 175.239 & 186.836 \\
$p$ & 0.000 & 0.003 & 0.000 & 0.000 & 0.000 \\
\hline
\end{tabular}

$S F F$, superior vertebral fat fraction; $I F F$, inferior vertebral fat fraction; $N P$, nucleus pulposus; $A A F$, anterior annulus fibrosus; $P A F$, posterior annulus fibrosus

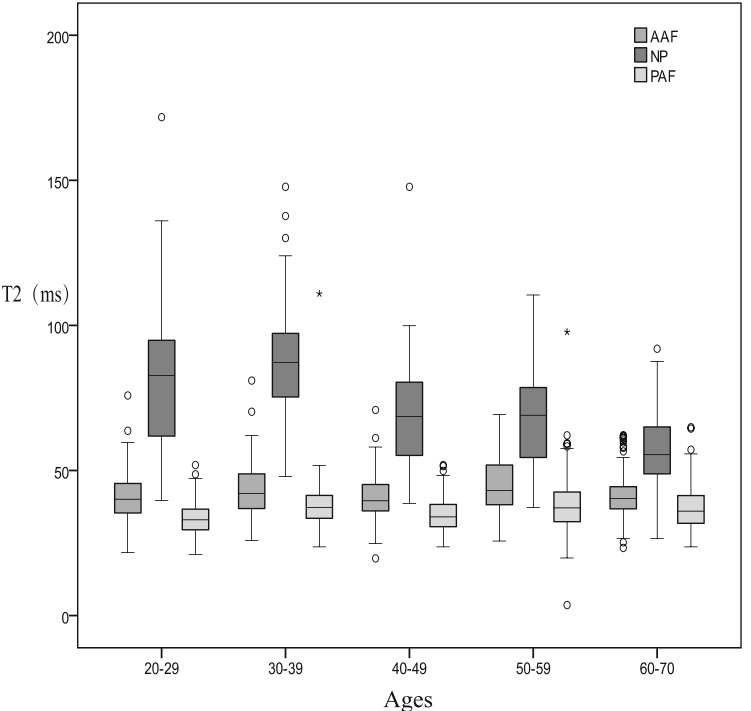

IFF, inferior vertebral fat fraction; NP, nucleus pulposus; AAF, anterior annulus fibrosus; PAF, posterior annulus fibrosus

between the early and the advanced degenerative groups. These results showed that it is the early stages of the IVDD and the BMF, and the IVDD can influence each other at some level.

According to the moderate correlation between Pfirrmann grading and VFF, the significance of VFF in both correlation analyses with the NP T2 value and discrimination between the advanced degenerated groups was minimal in our study and may limit the clinical relevance. The small sample size of Pfirrmann IV (12.7\%) with severe IVDD might be a possible explanation. Since IVDD and BMF may be affected by age as well as BMI, the presence of elderly or overweight individuals may have influenced the results. In Pfirrmann V, we were unable to determine whether there was a correlation or not due to the measurement error associated with collapsed disc height.

A moderate to high inverse correlation has been observed between the BMF (mean and SD) and T1 $\rho / \mathrm{T} 2$ (mean and SD) in the adjacent disc of most lumbar levels by Vangeneugden et al. [27]. Similarly, they also have reported a significant correlation between BMF SD and adjacent disc Pfirrmann classification, with a correlation coefficient of 0.53 [27]. We reconfirmed that the severity of disc degeneration increased with the increase of fat in adjacent vertebral bodies, and this relationship is more pronounced in L4/5 lumbar levels. The number of IVDD occurring alone in one segment was highest in the L4/5, suggesting that L4/5 IVD may be the most vulnerable segment. Fullerenol nanoparticles, as free radical scavengers, may prevent vertebral fatty marrow deposition and inflammatory responses during disc degeneration [28]. Disc height is associated with increased vertebral fracture risk in postmenopausal women [13]. The BMF can, therefore, 


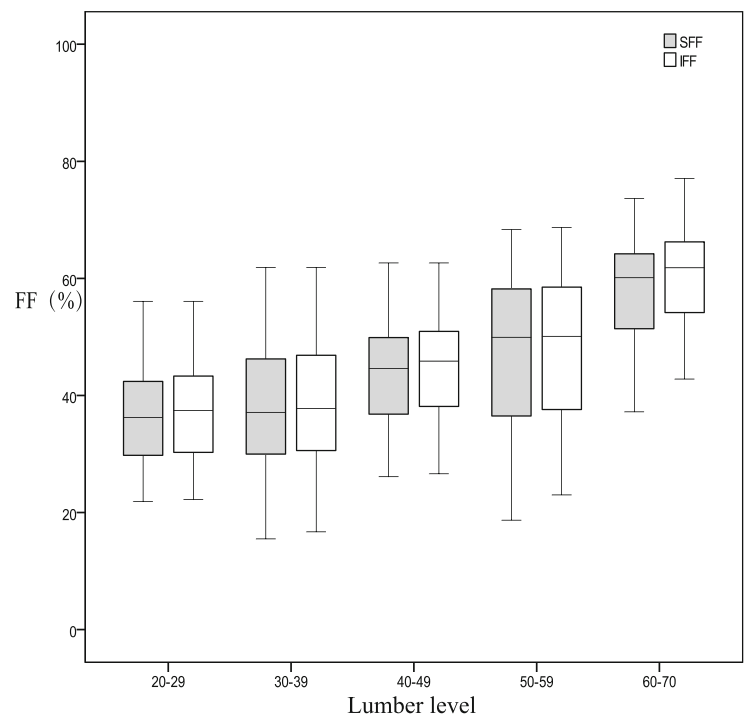

Fig. 8 Different lumbar levels of disc nucleus pulposus and annulus fibrosus T2 values of the inferior and superior adjacent vertebral body $\mathrm{FF}$ values. The $\mathrm{T} 2$ value decreased and the $\mathrm{FF}$ value increased from $\mathrm{L} 1$ to

directly or indirectly change the disc's microstructure and its biochemical composition. This study confirmed the role of vertebral BMF in lumbar disc degeneration and provides further evidence in the currently limited field.

MRI quantitative and semi-quantitative studies have been conducted for patients with various ages with a primary focus on the variation of IVDD [29] or BMF [30], modic changes [31], and T2 and FF parameters studied separately with increasing age. To our knowledge, most studies have not reported the

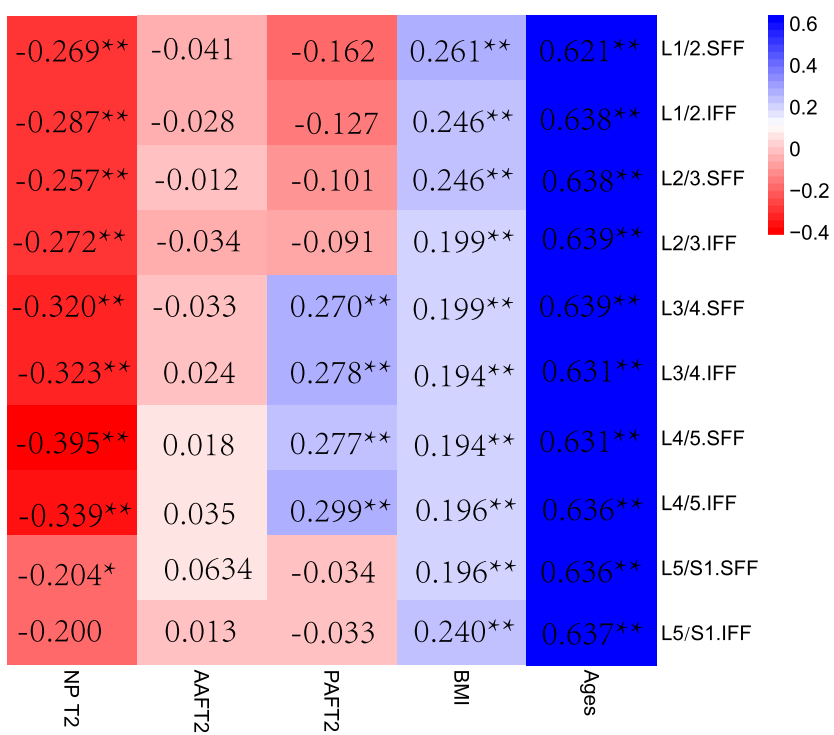

Fig. 9 Correlations of FF with NP T2 values, annulus fibrosus T2 values, BMI, and ages in different lumbar levels. $* p<0.05$; $* p<0.01$. Values marked with an asterisk indicate statistical significance. FF, fat fraction; $\mathrm{SFF}$, superior vertebral fat fraction; IFF, inferior vertebral fat fraction; $\mathrm{NP}$, nucleus pulposus; AAF, anterior annulus fibrosus; PAF, posterior annulus fibrosus

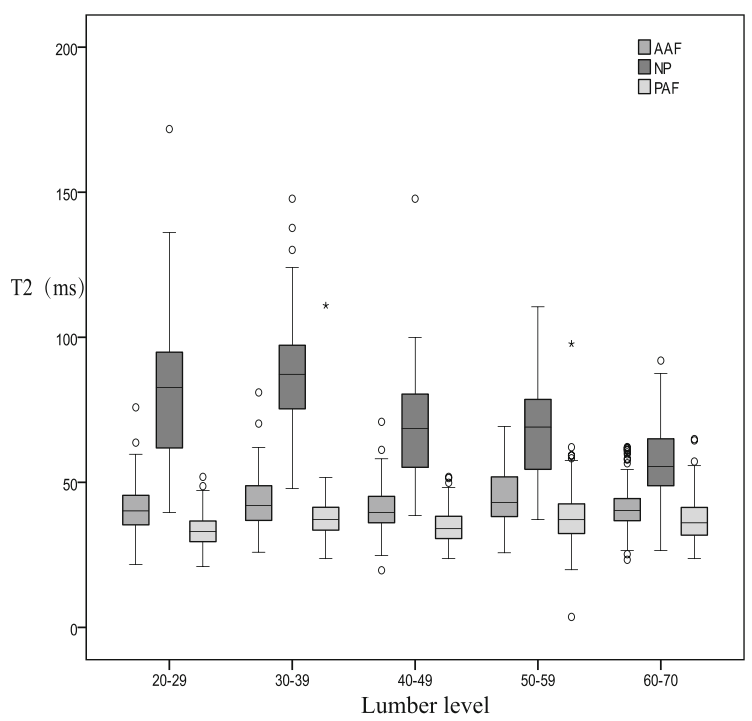

L5 levels. FF, fat fraction; SFF, superior vertebral fat fraction; IFF, inferior vertebral fat fraction; NP, nucleus pulposus; AAF, anterior annulus fibrosus; PAF, posterior annulus fibrosus

correlation of IVD T2 value and the Pfirrmann grading parameters with BMF in different ages. In age groups 40-49, NP T2 value was found to be weakly inversely correlated with adjacent BMF, while other age groups appeared to demonstrate no statistically significant correlation. This indicates that the most rapidly changing phase (the disc degeneration and fat conversion speed) is the same as the relationship between Pfirrmann II-III.

Our study demonstrated significant associations between the biochemical degeneration of IVD and adjacent vertebrae BMF using advanced MRI quantitative techniques such as IDEAL-IQ and T2 mapping, as well as MRI biomarkers sensitive and objective to collagen integrity and water content of the disc [32]. We found some compositional changes in the disc and bone marrow, which occurred earlier than the visually observable morphologic changes with conventional MRI

Table 6 Differences of VFF and T2 in AAF, NP, and PAF in the five lumbar levels

\begin{tabular}{llllll}
\hline \multirow{2}{*}{\multicolumn{1}{l}{ T2 }} & \multicolumn{4}{c}{ SFF } & IFF \\
\cline { 2 - 4 } & NP & AAF & PAF & & \\
\hline L1/2 & $72.6 \pm 19.3$ & $40.3 \pm 8.4$ & $39.0 \pm 12.3$ & $40.1 \pm 11.3$ & $43.3 \pm 12.4$ \\
L2/3 & $76.0 \pm 20.4$ & $43.9 \pm 9.5$ & $37.5 \pm 6.3$ & $43.2 \pm 12.5$ & $45.6 \pm 12.8$ \\
L3/4 & $76.6 \pm 20.4$ & $45.3 \pm 9.0$ & $37.0 \pm 6.5$ & $45.6 \pm 12.7$ & $45.4 \pm 12.7$ \\
L4/5 & $72.3 \pm 21.5$ & $44.2 \pm 8.8$ & $35.4 \pm 6.1$ & $44.8 \pm 12.9$ & $45.4 \pm 12.9$ \\
L5/S1 & $67.6 \pm 20.6$ & $38.4 \pm 8.2$ & $33.0 \pm 7.2$ & $46.2 \pm 12.9$ & $44.6 \pm 13.1$ \\
$\chi 2$ & 12.184 & 45.154 & 36.957 & 13.601 & 1.900 \\
$p$ & 0.016 & 0.000 & 0.000 & 0.006 & 0.754 \\
\hline
\end{tabular}

$S F F$, superior vertebral fat fraction; $I F F$, inferior vertebral fat fraction; $N P$, nucleus pulposus; $A A F$, anterior annulus fibrosus; $P A F$, posterior annulus fibrosus 
sequences. Consequently, quantitative measurement of vertebral BMF content has potential predictive value in early disc degeneration.

Our study had several limitations. First, our study is a cross-sectional cohort study, and a longitudinal study needs to be carried out to obtain further evidence. Second, our results may be limited since it was only based on the relationship between T2 mapping and BMF, and perfusion factors associated with cellular and microvascular density were not evaluated. However, Karampinos et al. confirmed that there is significant correlation between reduced vertebral bone marrow perfusion indices and osteoporosis using dynamic contrastenhanced MRI [33]. Additionally, transversal T2 mapping scanning with a 3-mm layer thickness may have various partial effects on the adjacent end plates due to disc space narrowing. Thus, adding a sagittal plane view in future studies may provide more information.

In conclusion, our results demonstrate that there is a close association between BMF and IVDD, and that a reciprocity relationship may exist between the vertebrae BMF and the disc nutrition supply. Novel quantitative measurement of BMF deposition and vertebral disc water content have potential predictive value and provide important supplements to the qualitative traditional classification strategies for the early stages of IVDD. However, the suggested causality needs to be further investigated and confirmed in larger longitudinal studies.

Funding information This grant of the study was from the National Natural Science Foundation of China (Grant No. 81572628) and the Guangdong Province Science and Technology plan project (Grant No. 2014A020212304). This funding made a significant contribution to study design, data interpretation, and writing.

\section{Compliance with ethical standards}

Conflict of interest The authors declare that they have no conflicts of interest.

Ethical approval All procedures performed in studies involving human participants were in accordance with the ethical standards of the institutional and/or national research committee and with the 1964 Helsinki declaration and its later amendments or comparable ethical standards.

Informed consent Informed consent was obtained from all individual participants included in the study.

IRB statement This study received institutional review board approval.

Open Access This article is licensed under a Creative Commons Attribution 4.0 International License, which permits use, sharing, adaptation, distribution and reproduction in any medium or format, as long as you give appropriate credit to the original author(s) and the source, provide a link to the Creative Commons licence, and indicate if changes were made. The images or other third party material in this article are included in the article's Creative Commons licence, unless indicated otherwise in a credit line to the material. If material is not included in the article's
Creative Commons licence and your intended use is not permitted by statutory regulation or exceeds the permitted use, you will need to obtain permission directly from the copyright holder. To view a copy of this licence, visit http://creativecommons.org/licenses/by/4.0/.

\section{References}

1. Freemont AJ. The cellular pathobiology of the degenerate intervertebral disc and discogenic back pain. Rheumatology (Oxford). 2009;48(1):5-10.

2. Ohtori S, Inoue G, Miyagi M, Takahashi K. Pathomechanisms of discogenic low back pain in humans and animal models. Spine J. 2015;15(6):1347-55.

3. Izzo R, Popolizio T, D’Aprile P, Muto M. Spinal pain. Eur J Radiol. 2015;84(5):746-56.

4. Modic MT, Ross JS. Lumbar degenerative disk disease. Radiology. 2007;245(1):43-61.

5. Lotz JC, Fields AJ, Liebenberg EC. The role of the vertebral end plate in low back pain. Glob Spine J. 2013;3(3):153-64.

6. Fathi Kazerooni A, Pozo JM, McCloskey EV, Saligheh Rad H, Frangi AF. Diffusion MRI for assessment of bone quality; a review of findings in healthy aging and osteoporosis. J Magn Reson Imaging. 2019.

7. De Bisschop E, Luypaert R, Louis O, Osteaux M. Fat fraction of lumbar bone marrow using in vivo proton nuclear magnetic resonance spectroscopy. Bone. 1993;14(2):133-6.

8. Kaiser J, Allaire B, Fein PM, Lu D, Jarraya M, Guermazi A, et al. Correspondence between bone mineral density and intervertebral disc degeneration across age and sex. Arch Osteoporos. 2018;13(1):123.

9. Wang YX, Griffith JF. Effect of menopause on lumbar disk degeneration: potential etiology. Radiology. 2010;257(2):318-20.

10. Castano-Betancourt MC, Oei L, Rivadeneira F, de Schepper EI, Hofman A, Bierma-Zeinstra S, et al. Association of lumbar disc degeneration with osteoporotic fractures; the Rotterdam study and meta-analysis from systematic review. Bone. 2013;57(1):284-9.

11. Fabreguet I, Fechtenbaum J, Briot K, Paternotte S, Roux C. Lumbar disc degeneration in osteoporotic men: prevalence and assessment of the relation with presence of vertebral fracture. J Rheumatol. 2013;40(7):1183-90.

12. Wang YXJ. Menopause as a potential cause for higher prevalence of low back pain in women than in age-matched men. J Orthop Transl. 2017;8:1-4.

13. Luo Y, Zhang L, Wang WY, Hu QF, Song HP, Su YL, et al. Alendronate retards the progression of lumbar intervertebral disc degeneration in ovariectomized rats. Bone. 2013;55(2):439-48.

14. Folman Y, Shabat S, Gepstein R. Relationship between low back pain in post-menopausal women and mineral content of lumbar vertebrae. Arch Gerontol Geriatr. 2004;39(2):157-61.

15. Griffith JF, Yeung DK, Tsang PH, Choi KC, Kwok TC, Ahuja AT, et al. Compromised bone marrow perfusion in osteoporosis. J Bone Miner Res. 2008;23(7):1068-75.

16. Meunier P, Aaron J, Edouard C, Vignon G. Osteoporosis and the replacement of cell populations of the marrow by adipose tissue. A quantitative study of 84 iliac bone biopsies. Clin Orthop Relat Res. 1971;80:147-54.

17. Moerman EJ, Teng K, Lipschitz DA, Lecka-Czernik B. Aging activates adipogenic and suppresses osteogenic programs in mesenchymal marrow stroma/stem cells: the role of PPAR-gamma2 transcription factor and TGF-beta/BMP signaling pathways. Aging Cell. 2004;3(6):379-89. 
18. Li CJ, Cheng P, Liang MK, Chen YS, Lu Q, Wang JY, et al. MicroRNA-188 regulates age-related switch between osteoblast and adipocyte differentiation. J Clin Invest. 2015;125(4):1509-22.

19. He J, Fang H, Li X. Vertebral bone marrow fat content in normal adults with varying bone densities at $3 \mathrm{~T}$ magnetic resonance imaging. Acta Radiol. 2019;60(4):509-15.

20. Kim H, Taksali SE, Dufour S, Befroy D, Goodman TR, Petersen $\mathrm{KF}$, et al. Comparative MR study of hepatic fat quantification using single-voxel proton spectroscopy, two-point dixon and three-point IDEAL. Magn Reson Med. 2008;59(3):521-7.

21. Aoki T, Yamaguchi S, Kinoshita S, Hayashida Y, Korogi Y. Quantification of bone marrow fat content using iterative decomposition of water and fat with echo asymmetry and least-squares estimation (IDEAL): reproducibility, site variation and correlation with age and menopause. Br J Radiol. 2016;89(1065):20150538.

22. Watanabe A, Benneker LM, Boesch C, Watanabe T, Obata T, Anderson SE. Classification of intervertebral disk degeneration with axial T2 mapping. AJR Am J Roentgenol. 2007;189(4):93642.

23. Mwale F, Iatridis JC, Antoniou J. Quantitative MRI as a diagnostic tool of intervertebral disc matrix composition and integrity. Eur Spine J. 2008;17(Suppl 4):432-40.

24. Alleva J, Hudgins T, Belous J, Kristin OA. Chronic low back pain. Dis Mon. 2016;62(9):330-3.

25. Pfirrmann CW, Metzdorf A, Zanetti M, Hodler J, Boos N. Magnetic resonance classification of lumbar intervertebral disc degeneration. Spine (Phila Pa 1976). 2001;26(17):1873-8.

26. Regier NG, Hodgson NA, Gitlin LN. Characteristics of activities for persons with dementia at the mild, moderate, and severe stages. Gerontologist. 2017;57(5):987-97.
27. Krug R, Joseph GB, Han M, Fields A, Cheung J, Mundada M, et al. Associations between vertebral body fat fraction and intervertebral disc biochemical composition as assessed by quantitative MRI. J Magn Reson Imaging. 2019;50(4):1219-26.

28. Liu Q, Jin L, Shen FH, Balian G, Li XJ. Fullerol nanoparticles suppress inflammatory response and adipogenesis of vertebral bone marrow stromal cells-a potential novel treatment for intervertebral disc degeneration. Spine J. 2013;13(11):1571-80.

29. Alvarez-Garcia O, Matsuzaki T, Olmer M, Masuda K, Lotz MK. Age-related reduction in the expression of FOXO transcription factors and correlations with intervertebral disc degeneration. J Orthop Res. 2017;35(12):2682-91.

30. Ricci C, Cova M, Kang YS, Yang A, Rahmouni A, Scott WW Jr, et al. Normal age-related patterns of cellular and fatty bone marrow distribution in the axial skeleton: MR imaging study. Radiology. 1990;177(1):83-8.

31. Tarukado K, Ono T, Tono O, Tanaka H, Ikuta K, Harimaya K, et al. Does modic change progresss with age? Spine (Phila Pa 1976). 2017;42(23):1805-9 doi:. (- 1528-1159 (Electronic)):- 1805-9.

32. Regatte RR, Akella SV, Borthakur A, Kneeland JB, Reddy R. Proteoglycan depletion-induced changes in transverse relaxation maps of cartilage: comparison of T2 and T1rho. Acad Radiol. 2002;9(12):1388-94.

33. Karampinos DC, Ruschke S, Dieckmeyer M, Diefenbach M, Franz D, et al. Quantitative MRI and spectroscopy of bone marrow. J Magn Reson Imaging. 2018;47(2):332-53. https://doi.org/10. 1002/jmri25769 Epub 2017. (- 1522-2586 (Electronic)):- 332-53.

Publisher's note Springer Nature remains neutral with regard to jurisdictional claims in published maps and institutional affiliations. 